\title{
Timing of the Vibration of Arm Muscles Affects Grip Force Control
}

\author{
by \\ Marcio J. Santos and Alexander S. Aruin ${ }^{1}$
}

The purpose of the study was to investigate how the timing of application of vibration to the arm muscles affects grip force. Eight healthy subjects performed similar tasks of lifting and holding an object without any vibration (NV) and with vibration applied to the extrinsic wrist and finger muscles at different times during the task: 1) applied immediately prior to the task performance $(A F V)$ and 2) during the task performance (DFV). Peak grip force, static grip force, and acceleration of the object were recorded. Vibration applied to the muscles during the task performance did not affect grip force generation. However, when vibration was applied prior to the task performance, a significant increase in grip force was observed. We suggest that the differences in magnitudes of grip force between the conditions are associated with the availability of information from muscle spindles and/or joint and tactile afferents. It appears that vibration applied during the task performance affects only muscles spindles, while a five-minute vibration applied prior to the lift of the object affects both muscle spindles and joint and tactile afferents. The results of the study provide additional information on the availability of afferent information in the control of grip force.

Key words: muscle vibration, hand, grip force control

Running Title: vibration of arm muscles and grip force

1 - Department of Physical Therapy, University of Illinois at Chicago, Chicago, IL 60612 


\section{Introduction}

Appropriate modulation of grip force is essential for the performance of various activities of daily living, such as drinking, eating, buttoning a shirt, etc. Proper modulation of grip force is also crucial in a number of work-related activities (Holewski et al. 1988; Bell-Krotoski 1991; Gilles and Wing 2003; Nowak and Hermsdorfer 2004). It is well established that a successful task of lifting an object using a finger grasp requires application of grip forces slightly above the object's mass in order to prevent slippage but not large enough to damage the object (Johansson and Cole 1994).

Earlier studies of grip force control focused on the importance of sensorimotor memory (internal models), which are based on long lasting previous experiences (Wolpert and Miall 1996), short-term memory, which depends on previous lifting (Nowak et al. 2004a), and knowledge of physical properties of the objects, i.e., object mass and density, friction between the fingers and the object, etc (Gordon et al. 1991). The physical properties of the object are perceived first by the object's visual analysis followed by information from the fingers' cutaneous mechanoreceptors (Jeannerod 1986; Johansson and Westling 1987; Johansson and Westling 1988; Gordon et al. 1991; Quaney et al. 2003). It seems that the central nervous system (CNS) controls grip force using feedforward and feedback commands (Johansson and Westling 1987; Gordon et al. 1991; Johansson and Cole 1992). In feedforward grip force control, grip forces are scaled in advance using internal models (sensorimotor memory) (Johansson and Cole 1992) established based on predictions of the physical characteristics of an object and the consequences of motor actions, such as movement trajectories of the grasping hand and its implications on object loading (Flanagan and Wing 1997) (Flanagan and Wing 1995; Blakemore et al. 1998). In feedback control, grip forces are finely adjusted during the task performance based on peripheral afferent signals. These signals are used to compare the actual and predicted forces: if a mismatch between them occurs, the motor command is corrected and the internal model is updated (Prochazka 1981; Miall 1996; Wolpert and Miall 1996).

For healthy individuals, the performance of one-two lifts is enough to update the internal models and generate adequate grip forces (Westling and Johansson 1984; Quaney et al. 2003). Such an efficient feedback information, which is crucial for modifying the ongoing movement and upgrading the system, relies mostly on the cutaneous receptors from the finger tips (Johansson and Cole 1994). However, it was demonstrated that there is a need for about 100 
ms after the first contact of the fingers with the object to utilize feedback tactile information and adjust the grip forces (Jenmalm and Johansson 1997).

Disruptions in tactile afferent signals from the finger tips severely impair grip force control (Johansson and Westling 1987; Nowak et al. 2003; Nowak and Hermsdorfer 2003a). For example, increased grip forces were seen in healthy individuals after digital anesthesia (Nowak et al. 2001; Augurelle et al. 2003) or cooling (Nowak and Hermsdorfer 2003a), as well as in individuals with impaired tactile sensibility (Nowak and Hermsdorfer 2003b). The results of these studies provided evidence in support of the importance of tactile afferent signals as a source of feedback information in the control of grip force.

Another way to disrupt utilization of feedback information from the fingers is the application of vibration to the muscles participating in grip force control. It was demonstrated recently that 10 minutes of vibration applied to the adductor pollicis and interosseus dorsalis muscles of the thumb (that excites primarily spindle receptors) caused a significant increase in the maximal grip force when lifting a familiar object (Nowak et al. 2004b). In another study, in which healthy individuals were instructed to match a target force magnitude shown on a computer screen, they applied smaller grip force when exposed to bilateral wrist vibration compared to no vibration conditions (Bock et al. 2005).

However, it is not clear whether these differences in the effect of vibration on grip force control relate to the differences in the experimental task. Or if they are due to differences in the way vibration was induced (before vs. simultaneously to the task performance), which may affect the afferent structures differently. For example, a long period of muscular vibration before the task might not only affect the muscle spindles but also joint and tactile receptors in the hand (Malchaire et al. 1998), which may impair grip force generation even more.

Several daily life tasks such as lifting an object, buttoning a short, or writing a letter involve utilization of the opposition of the thumb and the flexion of the other 4 fingers. The extrinsic muscles that control flexion of the wrist and stability of finger joints are crucial in grip force generation (Long 1968). In fact, muscular forces that drive flexion of the distal and middle phalanges of the fingers rely mostly on extrinsic flexor muscles of the hand (Flexor digitorum superficialis and profundus) (Li et al. 2000). In addition, these muscles are either commonly involved in spastic syndromes (cerebral palsy or post stroke) or their innervations (median and ulnar nerves) are frequently implicated in compressive syndromes (Bora and Osterman 1982). Therefore, it is important to study how extrinsic flexor muscles react to applied vibrations. 
The purpose of this study was to investigate how vibration of the wrist and finger flexors affects grip force control during performance of a common task of lifting and holding an object. We hypothesized that grip force will increase with application of vibration prior the task, but it will not increase if vibration is applied during the task performance.

\section{Methods}

\section{Subjects}

Eight subjects ( 5 females and 3 males, mean age $30 \pm 6.7$ years) participated in the study. They were healthy volunteers with no history of any neurological illness or musculoskeletal disorder that could impair motor performance with their hands. All subjects signed an informed consent form that was approved by the Institutional Review Board of the University of Illinois at Chicago.

\section{Apparatus}

A cylindrical plastic cup (diameter, $6 \mathrm{~cm}$; height, $17 \mathrm{~cm}$; total weight of 0.340 $\mathrm{Kg}$ ) was used in the study. The cup was instrumented with a strain gauge (model 208C03, PCB Piezotronics, USA), which was located on the side of the cup at a distance of $8 \mathrm{~cm}$ from the cup's bottom, and an accelerometer (model 333b32, PCB Piezotronics, USA) attached to the cup at its midpoint. The force sensor measured the grip force applied to the cup by the thumb and opposing four fingers. The accelerometer was used to measure the vertical acceleration of the cup.

A custom-made vibrator $(2.5 \mathrm{~cm}$ in diameter and $5 \mathrm{~cm}$ long) covered with a plastic shield was used to induce muscular vibrations. The 6 VDC motor (Mabuche RE-14, Mabuche Motor CO., LTD; Japan) with a small cylindrical metal pebble $(0.014 \mathrm{Kg})$ asymmetrically attached to its axis produced vibrations with a frequency of $80 \mathrm{~Hz}$ and amplitude of $1 \mathrm{~mm}$.

\section{Procedure}

The subjects were seated upright in an adjustable chair with both feet flat and supported on the floor. After washing (with soap and water) and drying their hands, the subjects were required to perform the experimental task that involved a vertical lift of the cup that was positioned on the table. The subjects were instructed to lift the cup, hold it for approximately $4 \mathrm{~s}$, and then place it back on the table. A $10 \mathrm{~cm}$ long marker was used to show the subjects the final position (Fig.1). The subjects also were instructed to lift the cup using primarily 
elbow flexion, keeping the forearm in a neutral position and the wrist slightly extended. Since the forearm did not touch the table surface during the experiment, a small movement in the shoulder joint was observed. The vibrator was firmly attached to the middle of the wrist and finger flexor muscle groups (two to three fingerbreadths volar to the ulna at the junction of the upper and middle thirds of the forearm) (Basmajian and Blumenstein 1980) with a hypoallergenic tape (Fig. 1). The subjects performed lifts with their dominant hand: seven subjects were right dominant and one subject was left dominant. Hand dominancy was assigned based on the subjects' hand preference for writing and grasping an object from the table.

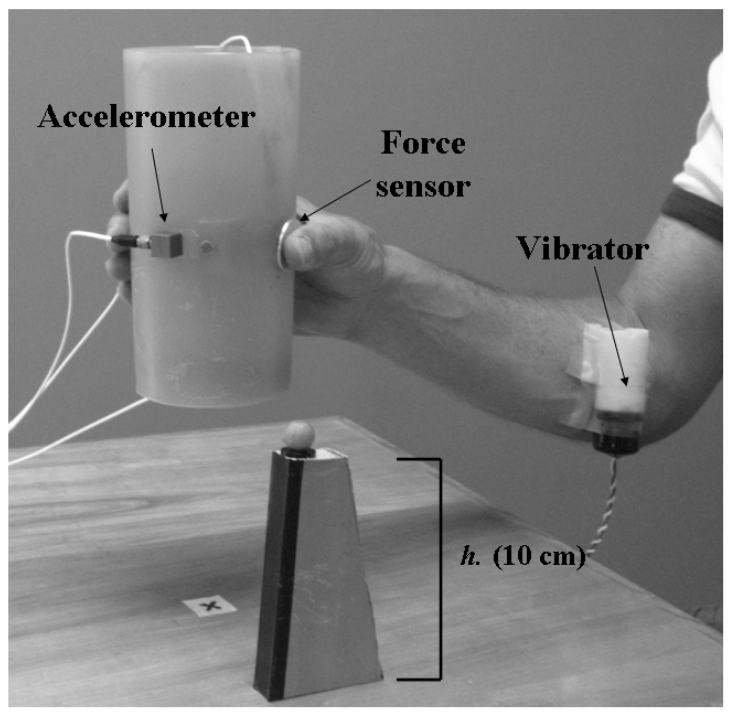

Fig. 1

The experimental setup. The task involves lifting and holding the plastic cup instrumented with a force cell and an accelerometer. $h$. is the height of lifts. The vibrator is attached to the forearm and allows application of vibration during and prior to the lift of the cup.

Three different experimental conditions were implemented. First, the subjects were instructed to lift the cup with no muscular vibration (NV). Second, they performed lifts of the cup when vibration was applied to the wrist flexors during the task performance (DFV). Third, the subjects performed the lifts of the cup after vibration was applied to the wrist flexors for 5 minutes (AFV). In each condition, the subjects performed 5 lifting trials. The vibrator was attached 
to the arm in all three conditions. However, it was turned off in the NV series and turned on just before the start of the first lift trial, remaining on during all 5 trials in DFV series. In the AFV series, the vibrator was on for five minutes when the subjects' arm rested on the table, and it was turned off just before the performance of the 5 lifting trials. The order of the NV and DFV series was randomized, however, the AFV series was always kept as the last condition to avoid the need for extended rest periods to eliminate the effects of a five-minute vibration. To avoid fatigue after each series, the subjects were provided with a rest period for at least 3 minutes, followed by a few active movements of wrist flexion and extension to minimize the vibration effects. They were instructed to lift the object at a natural and comfortable velocity and to keep a similar velocity of arm movement across the series. The subjects practiced 3 to 5 lifting trials before the actual experiment began. No vibration was applied during practice trials. A few subjects reported an itchy but tolerable sensation during the muscular vibration.

\section{Data processing}

The signals from the force sensor and accelerometer were sampled at $100 \mathrm{~Hz}$ with a 16-bit resolution using LabView software and stored for off-line processing on a computer. After filtering (second order Butterworth filter with a cutoff frequency of $20 \mathrm{~Hz}$ ), the signals were analyzed using an interactive MATLAB program. The following measures were obtained: 1) Peak grip force (PGF), as a maximum grip force applied by the thumb during the task performance $(\mathrm{N}) ; 2)$ a maximum acceleration during the task performance $\left(\mathrm{AC}_{\max }\right)$ $\left.\left(\mathrm{m} / \mathrm{sec}^{2}\right) ; 3\right)$ static force $(\mathrm{SF})$, a mean of grip force measured during a $1.5 \mathrm{~s}$ time window during lifting the object after the acceleration becomes zero; 4) force ratio (PGF/ LF max) between the PGF and maximal load force (LFmax) that describes the efficiency of produced grip force; 5) time lag between the PGF and $\mathrm{AC}_{\max }$ (TPGF-AC). In addition, PGF measures were compared between the trials in each experimental condition to evaluate the effects of vibration in short-term memory during the 5 lifting trials. Since 1 or 2 lifting trials are necessary to adjust the grip forces during natural conditions, we compared the PGF means of the first two trials with the PGF means of the last 3 trials.

\section{Statistical analysis}

A repeated measures ANOVA was performed with within-subject factors of conditions (NV, WFV, and AFV). The dependent variables were PGF, $\mathrm{AC}_{\max }, \mathrm{ST}$, PGF/LFmax, and TPGF-Ap. Once a significant difference was found for these vari- 
ables, a post-hoc analysis was performed to detect which pairs were significantly different. Paired T-tests were performed to compare the PGF means of the first 2 lifting trials with the means of the last 3 lifting trials for all three experimental conditions. The $p$ value was set at 0.05 level for all statistical tests.

\section{Results}

The vibration applied to the wrist flexors did not alter the movement performance as the subjects were able to execute the tasks using similar acceleration. Thus, the object acceleration $\left(\mathrm{AC}_{\max }\right)$ was $2.04 \pm 0.1 \mathrm{~m} / \mathrm{s}^{2}$ for the NV condition. For the DFV and ADV conditions, the magnitudes of $A C_{\max }$ were $1.99 \pm 0.12$ $\mathrm{m} / \mathrm{s}^{2}$ and $1.89 \pm 0.18 \mathrm{~m} / \mathrm{s}^{2}$, respectively. The difference between $A C_{\max }$ across conditions was not statistically significant.

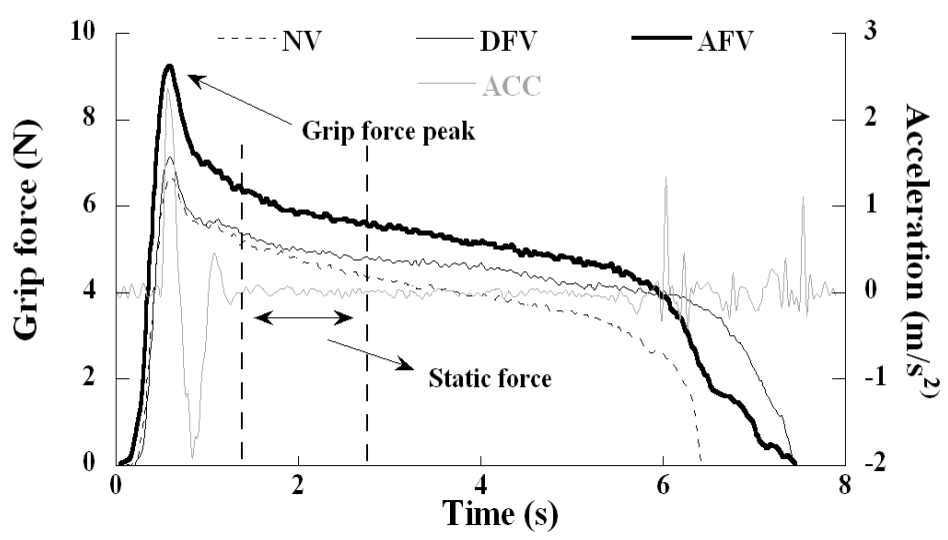

Fig. 2

Grip force (left axis) and acceleration (right axis) traces averaged across the five trials for a representative subject performing experimental tasks of lifting and holding an object with no vibration (NV), during (DFV), and after (AFV) wrist flexor vibrations. Notice that both peaks of grip force and static force were increased in the AFV condition. The acceleration trace is shown for the AFV condition.

Fig. 2 shows traces of grip forces for a representative subject recorded during the three experimental conditions (NV, DFV, and AFV). Notice that grip force traces are quite similar in the NV and DFV conditions. However, grip force trace is substantially higher in the AFV condition. The mean PGF in NV and DFV conditions were $8.67 \pm 2.9 \mathrm{~N}$ and $8.86 \pm 3.7 \mathrm{~N}$, respectively, while it increased in the AFV condition reaching 10.31 $\pm 3.6 \mathrm{~N}$. The ANOVA analysis 
showed significant differences in PGF across the three experimental conditions $(p=0.04)$. The post hoc analysis revealed that PGF was significantly greater for the AFV than for the NV condition $(p<0.01)$ (Fig. 3).

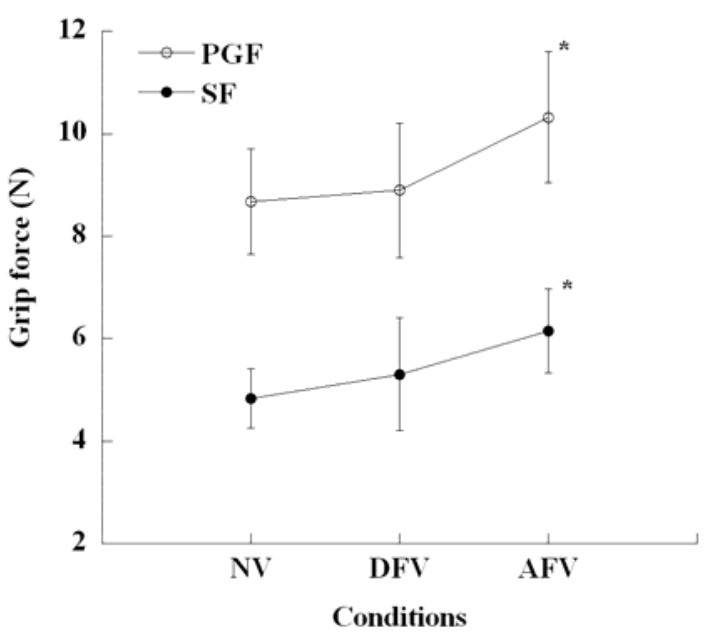

Fig. 3

Peak grip force (PGF) and static force (ST) during the three experimental conditions.

The abbreviations are explained in Fig 2. ${ }^{*}$ denotes significant difference at $p<0.05$

level.

The static force (SF) was $4.8 \pm 1.6 \mathrm{~N}$ and $5.3 \pm 3.1 \mathrm{~N}$ in the NV and DVF conditions, and it increased to $6.16 \pm 2.3 \mathrm{~N}$ in the AFV condition. Although the difference across conditions was not statistically significant $(p=0.19)$, post hoc analysis detected significant differences between the NV and AFV conditions $(p=$ 0.01 ).

The PGF/LFmax was $1.7 \pm 0.7$ in the NV condition, and it was $1.8 \pm 0.9$ and 2.1 \pm 0.9 in the DVF and AFV conditions, respectively. The difference in PGF/LF $\max$ between conditions was statistically significant $(p=0.02)$. PGF/LF max in NV conditions was significantly smaller than the PGF/LF $\max$ in the AFV condition detected by the post hoc analysis $(p<0.01)$.

The TPGF-AC was $108 \pm 27,107 \pm 27$ and $119 \pm 57 \mathrm{~ms}$ for NV, AFV and AFV, respectively. There was no significant difference between TPGF-AC across the conditions.

The results of analysis of the PGF during performance of the five trials revealed that there were no significant differences between the mean of the first 2 
trials and the mean of the last 3 lifting trials for all conditions (NV, DFV, and AFV).

\section{Discussion}

The first principal finding of this study is that short term vibration of the wrist flexors applied prior to lifting of the object affected the grip force control. Thus, peak grip force, static force, and the ratio between grip and load forces increased significantly after 5 minutes of muscular vibration compared to the condition with no vibration.

Why was the vibration preceding the task of lifting the object associated with higher grip forces? There are two possible explanations for this finding. First, it is known that efficient grip force control depends on utilization of shortterm memory related to the lifting of the object obtained in the previous practice trials. Thus, increase in grip force observed after the five minute vibration could be due to a vibration-related disruption of the short term memory used for scaling grip forces. Such a possibility was described in the literature. For example, the results of a recent study involving vibration of the adductor pollicis and interosseus dorsalis muscles of the thumb prior to the lifting task pointed out a possibility of disruption of the short term memory (Nowak et al. 2004b). However, there were important differences between experimental conditions in the later and the current study. Thus, vibration was applied to different muscles: adductor pollicis and interosseus dorsalis, which are the intrinsic and extrinsic wrist flexor muscles. In addition, the vibration application time was different (10 vs. $5 \mathrm{~min}$ ), as well as the grip type used in the two studies (the opposition of the thumb and index finger vs. the opposition of the thumb and the rest of fingers). Nevertheless, grip force increased after vibration in both studies, suggesting that most likely the CNS was not able to utilize short-term memory related to lifting the object. Second, five minutes of forearm vibration may alter the sensitivity of the tactile receptors, thus, affecting the way feedback information about the task performance is obtained. For example, through the analysis of the vibration perception threshold of the finger tip using a modified audiometer, it was demonstrated that both FA-I and -II mechanoreceptors at the finger tip were strongly affected after short term hand-arm vibrations (Malchaire et al. 1998). These authors also observed considerable paresthesia in the finger tips after only a few minutes of vibration (2-4 min). It is important to mention that a few subjects in a previous study involving vibration of the adductor pollicis and interosseus dorsalis muscles of the thumb reported numbness sensations in the fingers (Nowak et al. 2004b). Moreover, in that study, vibration was applied very close to the finger tips (especially the tip of the 
thumb). Thus, it is possible that even without apparent numbness sensation, a few minutes of forearm vibration may be sufficient to disrupt the cutaneous afferent inputs from the finger tips, thus affecting the grip force control in the past (Nowak et al. 2004b) and the present study. A likelihood of such a scenario is supported by well studied increases of grip force as a result of fingers' anesthesia (Nowak et al. 2001).

Another notable finding is related to changes in the ratio of grip and load force (PGF/LFmax). The ratio represents the coupling of both forces that form the basis for an efficient control of grasping (Hager-Ross and Johansson 1996). PGF/LF max in the current study statistically significantly increased after five minutes of vibration in the AFV condition compared to the NV condition. Similar increases of PGF/LFmax was observed in healthy individuals after fingers' anesthesia (Augurelle et al. 2003) and in patients with impaired tactile afferents (Nowak and Hermsdorfer 2003b).

A third important finding is that when the task was accompanied with vibration of the muscles, the subjects were able to maintain their grip force at the same level as without vibration. It seems that because the time of vibration was significantly shorter in this part of the study ( $\sim 5 \mathrm{sec}$ was needed to perform the 5 lifting trials), vibration most likely did affect muscle spindles but had no effect on the tactile receptors, as could happen in case of a significantly longer vibration. Thus, one could suggest that because the afferent inputs from the extrinsic muscles of the wrist and the flexors of the fingers did not affect the grip force control; it is possible that the CNS is able to pre-set an adequate force production without taking into consideration information from the extrinsic muscles. This suggestion is supported by previous findings that forearm and hand muscle and tendon afferents respond only after grip force starts to increase during tangential forces applied to a gripped manipulandum (Malchaire et al. 1998). This suggests that muscle and tendon afferents do not participate in the initial scaling of grip forces. Instead, they may provide information about joint torques during the ongoing tasks (Macefield and Johansson 1996). Although, the task used in the present study was different (simply hold a cup in stationary position), the individuals also seem to not rely on the information from the tendon and muscle afferents to scale PGF and adjust static grip forces (ST). It is likely that the feedback information arose from other available sources, such as joint and tactile receptors.

Therefore, it seems reasonable to suggest that five minutes of wrist and fingers vibration affected the muscle spindles along with other afferents, i.e., joint and mostly tactile afferents, which resulted in a considerable decrease of the efficiency of grip force generation. In contrast, when the vibration was applied 
during the task, it also affected the muscle spindles but did not influence the tactile afferents; as a result, grip force control was not affected.

There are other factors that can play a role in the observed changes in grip force in the presence of muscle vibration or after vibration. Thus, it is known that vibration can induce illusory movements (Casini et al. 2006; Knox et al. 2006; Courtine et al. 2007; Naito et al. 2007). For example, vibration of the wrist muscle tendon excites muscle spindle afferents that signal hand movement (Naito and Ehrsson 2006). The way illusory movements are induced depends on how vibration is applied to the hand. For example, when people felt illusory flexion of the arm while the hand was placed on the top of a cylinder, they experienced the cylinder shrinking along with the illusory arm movement (Naito et al. 2002; Naito and Ehrsson 2006). On the other hand, vibrating the forearm fixed on a static wall while participants were seated on a rotatable chair was associated with feeling rotation of the entire body (Lackner 1988). These results suggest that differences in the application of vibration in our experiments (during the arm movement vs. arm on the top of the table) might influence the way vibration is perceived.

In conclusion, a short period of wrist flexor muscular vibrations might be enough to affect the grip force control via decreased cutaneous sensibility or by affecting short term memory. In contrast, scaling and adjusting the grip force do not seem to rely on afferent inputs from the wrist flexors since they might be the principal inputs affected by the vibration applied during the performance of the lifting tasks.

\section{References}

Augurelle A.S., Smith A.M., Lejeune T., Thonnard J.L. (2003) Importance of cutaneous feedback in maintaining a secure grip during manipulation of hand-held objects. J Neurophysiol 89: 665-671

Basmajian J.V., Blumenstein R. (1980) Electrode placement in EMG biofeedback. Williams \& Wilkins, Baltimore

Bell-Krotoski J. (1991) Advances in sensibility evaluation. Hand Clin 7: 527-546

Blakemore SJ, Goodbody SJ, Wolpert DM (1998) Predicting the consequences of our own actions: the role of sensorimotor context estimation. J Neurosci 18: 7511-7518

Bock O., Vercher J.L., Gauthier G. (2005) Wrist vibration affects the production of finely graded forces. Aviat Space Environ Med 76: 435-440

Bora F.W, Jr., Osterman A.L. (1982) Compression neuropathy. Clin Orthop Relat Res: 20-32 
Casini L., Romaiguere P, Ducorps A, Schwartz D, Anton JL, Roll JP (2006) Cortical correlates of illusory hand movement perception in humans: a MEG study. Brain Res 1121: 200-206

Courtine G., De Nunzio A.M., Schmid M., Beretta M.V., Schieppati M. (2007) Stance- and locomotion-dependent processing of vibration-induced proprioceptive inflow from multiple muscles in humans. J Neurophysiol 97: 772-779

Flanagan J.R., Wing A.M. (1995) The stability of precision grip forces during cyclic arm movements with a hand-held load. Exp Brain Res 105: 455-464

Flanagan J.R., Wing A.M. (1997) The role of internal models in motion planning and control: evidence from grip force adjustments during movements of hand-held loads. J Neurosci 17: 1519-1528

Gilles M.A., Wing A.M. (2003) Age-related changes in grip force and dynamics of hand movement. J Mot Behav 35: 79-85

Gordon A.M., Forssberg H., Johansson R.S., Westling G. (1991) Integration of sensory information during the programming of precision grip: comments on the contributions of size cues. Exp Brain Res 85: 226-229

Hager-Ross C., Johansson R.S. (1996) Nondigital afferent input in reactive control of fingertip forces during precision grip. Exp Brain Res 110: 131141

Holewski J.J., Stess R.M., Graf P.M., Grunfeld C. (1988) Aesthesiometry: quantification of cutaneous pressure sensation in diabetic peripheral neuropathy. J Rehabil Res Dev 25: 1-10

Jeannerod M. (1986) The formation of finger grip during prehension. A cortically mediated visuomotor pattern. Behav Brain Res 19: 99-116

Jenmalm P., Johansson R.S. (1997) Visual and somatosensory information about object shape control manipulative fingertip forces. J Neurosci 17: 4486-4499

Johansson R.S., Cole K.J. (1992) Sensory-motor coordination during grasping and manipulative actions. Curr Opin Neurobiol 2: 815-823

Johansson R.S., Cole K.J. (1994) Grasp stability during manipulative actions. Can J Physiol Pharmacol 72: 511-524

Johansson R.S., Westling G. (1987) Significance of cutaneous input for precise hand movements. Electroencephalogr Clin Neurophysiol Suppl 39: 53-57

Johansson R.S, Westling G. (1988) Coordinated isometric muscle commands adequately and erroneously programmed for the weight during lifting task with precision grip. Exp Brain Res 71: 59-71 
Knox J., Cordo P., Skoss R., Durrant S., Hodges P. (2006) Illusory changes in head position induced by neck muscle vibration can alter the perception of elbow position. Behav Neurosci 120: 1211-1217

Lackner J.R. (1988) Some proprioceptive influences on the perceptual representation of body shape and orientation. Brain 111 ( Pt 2): 281-297

Li Z.M., Zatsiorsky V.M., Latash M.L. (2000) Contribution of the extrinsic and intrinsic hand muscles to the moments in finger joints. Clin Biomech (Bristol, Avon) 15: 203-211

Long C., 2nd (1968) Intrinsic-extrinsic muscle control of the fingers. Electromyographic studies. J Bone Joint Surg Am 50: 973-984

Macefield V.G., Johansson RS (1996) Control of grip force during restraint of an object held between finger and thumb: responses of muscle and joint afferents from the digits. Exp Brain Res 108: 172-184

Malchaire J., Rodriguez Diaz L.S., Piette A., Goncalves Amaral F., de Schaetzen D. (1998) Neurological and functional effects of short-term exposure to hand-arm vibration. Int Arch Occup Environ Health 71: 270-276

Miall RC (1996) Task-Dependent Changes in Visual Feedback Control: A Frequency Analysis of Human Manual Tracking. J Mot Behav 28: 125-135

Naito E., Ehrsson H.H. (2006) Somatic sensation of hand-object interactive movement is associated with activity in the left inferior parietal cortex. J Neurosci 26: 3783-3790

Naito E., Kochiyama T., Kitada R., Nakamura S., Matsumura M., Yonekura Y., Sadato N. (2002) Internally simulated movement sensations during motor imagery activate cortical motor areas and the cerebellum. J Neurosci 22: 3683-3691

Naito E., Nakashima T., Kito T., Aramaki Y., Okada T., Sadato N. (2007) Human limb-specific and non-limb-specific brain representations during kinesthetic illusory movements of the upper and lower extremities. Eur J Neurosci 25: 3476-3487

Nowak D.A., Glasauer S., Hermsdorfer J. (2003) Grip force efficiency in longterm deprivation of somatosensory feedback. Neuroreport 14: 1803-1807

Nowak D.A., Glasauer S., Hermsdorfer J. (2004a) How predictive is grip force control in the complete absence of somatosensory feedback? Brain 127: 182-192

Nowak D.A., Hermsdorfer J. (2003a) Digit cooling influences grasp efficiency during manipulative tasks. Eur J Appl Physiol 89: 127-133 
Nowak D.A., Hermsdorfer J. (2003b) Selective deficits of grip force control during object manipulation in patients with reduced sensibility of the grasping digits. Neurosci Res 47: 65-72

Nowak D.A., Hermsdorfer J. (2004) [Analysis of grip force during object manipulation. Method for the objective measurement of physiological normal and impaired hand function]. Nervenarzt 75: 725-733

Nowak D.A., Hermsdorfer J., Glasauer S., Philipp J., Meyer L., Mai N. (2001) The effects of digital anaesthesia on predictive grip force adjustments during vertical movements of a grasped object. Eur J Neurosci 14: 756-762

Nowak D.A., Rosenkranz K., Hermsdorfer J., Rothwell J. (2004b) Memory for fingertip forces: passive hand muscle vibration interferes with predictive grip force scaling. Exp Brain Res 156: 444-450

Prochazka A. (1981) Muscle spindle function during normal movement. Int Rev Physiol 25: 47-90

Quaney B.M., Rotella D.L., Peterson C., Cole K.J. (2003) Sensorimotor memory for fingertip forces: evidence for a task-independent motor memory. J Neurosci 23: 1981-1986

Westling G., Johansson R.S. (1984) Factors influencing the force control during precision grip. Exp Brain Res 53: 277-284

Wolpert D.M., Miall R.C. (1996) Forward Models for Physiological Motor Control. Neural Netw 9: 1265-1279

\section{Corresponding author:}

\section{Marcio J. Santos, Ph.D.}

Department of Physical Therapy (MC 898)

University of Illinois at Chicago

1919 W Taylor St (4th floor)

Chicago, IL 60612

E-mail: santosmj@uic.edu

Phone: (312) 355- 0902

Fax: (312) 996-4583

Authors submited their contribution of the article to the editorial board.

Accepted for pinting in Journal of Human Kinetics vol. 19/2008 on March 2008. 